\title{
MEASUREMENT OF THE LONGITUDINAL MICROWAVE INSTABILITY IN THE APS STORAGE RING*
}

\author{
Y.-C. Chae ${ }^{\dagger}$, L. Emery, A.H. Lumpkin, J. Song, B.X. Yang \\ Advanced Photon Source, Argonne National Laboratory, Argonne, IL
}

\begin{abstract}
We studied a longitudinal, single-bunch instability in the Advanced Photon Source (APS) storage ring. Previous measurements showed significant bunch lengthening without a comparable growth in energy spread [1]. Because the small dispersion at a bending magnet source point made it difficult to resolve the small change in the energy spread, we adjusted the lattice to make a dispersion bump within a sector, providing a large dispersion at the source point. The enhanced component from dispersion allowed us to measure the energy spread with greater accuracy. We observed anomalous growth in the energy spread beyond a threshold current of $7.2 \mathrm{~mA}$ with an rf voltage of $9.4 \mathrm{MV}$. The energy spread increased from 9.0E-4 to $9.8 \mathrm{E}-4$ above the threshold current up to $10 \mathrm{~mA}$. We also observed the appearance of the coherent signal at four times the synchrotron frequency beyond the threshold current, which indicates that the growth in energy spread is caused by a longitudinal instability.
\end{abstract}

\section{INTRODUCTION}

Measurement of the bunch length and horizontal beam size at a bending magnet source point in the Advanced Photon Source (APS) 7-GeV storage ring was conducted during the APS commissioning period; the results were published in [1]. At that time, we attained up to $18 \mathrm{~mA}$ in a single bunch, which corresponds to $400 \mathrm{~A}$ in peak current. Significant bunch lengthening following the 1/3power law was measured by a streak camera. However, the energy spread measured by the horizontal beam-size measurement at a non-zero dispersion location did not show the significant increase that the microwave instability theory predicts.

Since then, small (8-mm) gap chambers were installed, and the transverse instability reduced the current limit down to about $10 \mathrm{~mA}$, depending on rf voltage. Under these conditions, we measured bunch length and energy spread versus single-bunch current again. Several improvements were noted: 1) the resolution of the $\mathrm{x}$-ray pinhole camera improved from $80 \mu \mathrm{m}$ to $22 \mu \mathrm{m}$, 2) the dispersion bump installed at the diagnostic sector made the beam size caused by the energy spread larger, 3) the power spectrum of the beam can now be simultaneously measured to identify any coherent motion associated with blow-up of longitudinal phase-space area.

\footnotetext{
*Work supported by U.S. Department of Energy, Office of Basic Energy Sciences, under Contract No. W-31-109-ENG-38.

†chae@aps.anl.gov
}

\section{DISPERSION BUMP}

The APS storage ring consists of 40 sectors. The tunes of the nominal lattices are $v_{x} / v_{y}=35.20 / 19.30$ respectively. We made a local dispersion bump at sector 35 , where the diagnostic insertion device (ID) is located. The lattice around sector 35 is shown in Fig. 1. Note the three source points, S35:BM, S35:ID, and S36:AM.

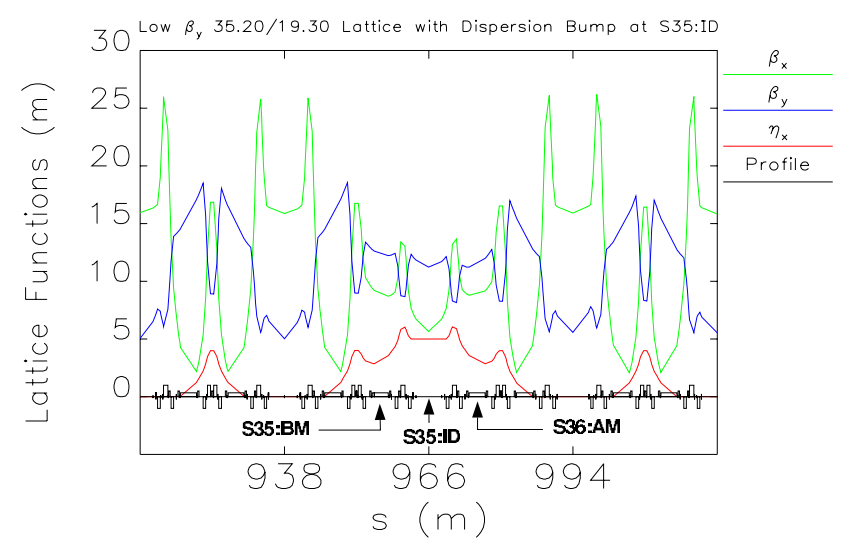

Figure 1: The lattice functions with dispersion bump at sector 35 on ID-straight.

Table 1: Comparison of Lattice Function at Source Points

\begin{tabular}{|c|c|c|c|c|}
\cline { 2 - 5 } \multicolumn{1}{c|}{} & \multicolumn{2}{c|}{ Nominal Lattice } & \multicolumn{2}{c|}{ Dispersion Bump } \\
\cline { 2 - 5 } \multicolumn{1}{c|}{} & $\beta_{\mathrm{x}}$ & $\eta_{\mathrm{x}}$ & $\beta_{\mathrm{x}}$ & $\eta_{\mathrm{x}}$ \\
\hline S35:BM & 1.70 & 0.08 & 8.72 & 0.28 \\
\hline S35:ID & 15.90 & 0.00 & 6.18 & 0.50 \\
\hline S36:AM & 4.38 & 0.001 & 8.11 & 0.34 \\
\hline
\end{tabular}

Table 2: Comparison of Source Size

\begin{tabular}{|c|c|c|c|c|}
\cline { 2 - 5 } \multicolumn{1}{c|}{} & \multicolumn{2}{c|}{ Nominal Lattice } & \multicolumn{2}{c|}{ Dispersion Bump } \\
\cline { 2 - 5 } \multicolumn{1}{c|}{} & $\sigma_{\beta}(\mu \mathrm{m})$ & $\sigma_{\mathrm{E}}(\mu \mathrm{m})$ & $\sigma_{\beta}(\mu \mathrm{m})$ & $\sigma_{\mathrm{E}}(\mu \mathrm{m})$ \\
\hline S35:BM & 116 & 80 & 264 & 280 \\
\hline S35:ID & 357 & 0 & 222 & 500 \\
\hline S36:AM & 198 & 1 & 255 & 340 \\
\hline
\end{tabular}

Table 1 compares the lattice functions of the source points. The data in Table 1 translate into the beam size comparison in Table 2 for a beam of emittance of $\varepsilon_{\mathrm{x}}=8$ $\mathrm{nm}$ and energy spread of $\delta_{\mathrm{E}}=0.1 \%$. We can see from Table 2 that only S35:BM, in the nominal lattice, can be used to measure energy spread via beam-size measurement. With the dispersion bump installed in the ring, however, we can use all three source points for the measurement. Also, because the bump is local and is made outside of the bending magnet, the effects on the longitudinal beam dynamics are minimal. 
When we first installed the dispersion bump in December 1999, we could store up to only $4 \mathrm{~mA}$ because of the global lattice perturbation caused by the bump, even though the tunes were corrected to the nominal values. In February 2000, we corrected the dispersion function by using the singular value decomposition (SVD) method [2]. The measured dispersion around the ring is shown in Fig. 2. The regular peak of $400 \mathrm{~mm}$ corresponds to the measurement at the center of the achromat, and the bump is shown near $\mathrm{s}=1,000 \mathrm{~m}$. Initial measurements of bunch length and energy spread have been carried out on this lattice.

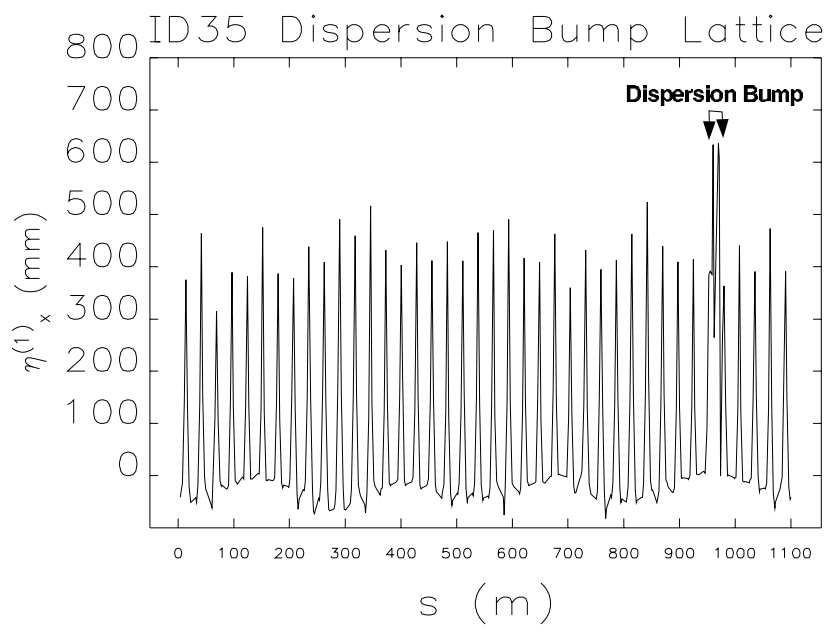

Figure 2: Measured dispersion function around ring.

\section{MEASUREMENT}

\subsection{Early Measurement with Dispersion Bump}

In March 2000, we measured bunch length and energy spread as functions of single-bunch current with the dispersion bump installed at sector 35 . Because of the small gap chamber, the maximum current we could store was about $10 \mathrm{~mA}$ in the single bunch. For bunch length measurement, we used the uv-visible streak camera to detect the light from S35:BM source points. However, for the beam size measurement, we used the image of the S36:AM source, in which the beam size is dominated by the energy spread. We took the data for different rf-gap voltages.

Typical data, with nominal rf voltage of $9.4 \mathrm{MV}$, are shown in Figs. 3 and 4. We reduced the current in small steps by pinging the beam into the horizontal scraper, after which we waited for a few radiation damping times before acquiring data from the optical instrument.

Bunch length data showed familiar power-law behavior; namely, varying as the 1/3-power of current. The data resemble either the anomalous bunch lengthening caused by microwave instability or the lengthening due to potential-well-distortion (PWD).

If the bunch lengthening was caused by microwave instability, we expected to observe a threshold current near $0.3 \mathrm{~mA}$. This value was predicted by using the Boussard criterion for the previously estimated impedance
$|\mathrm{Z} / \mathrm{n}|_{0}=0.5 \Omega$. With bunch lengthening data alone, it is difficult to determine where the threshold is.

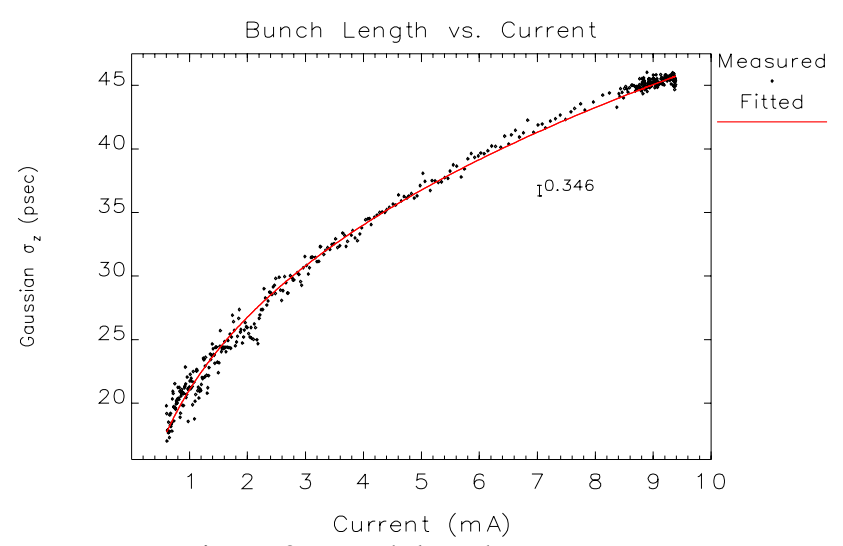

Figure 3: Bunch length vs. current.

However, the threshold is clearly shown in Fig. 4, where the energy spread versus current is depicted in three different regimes. Region-I is up to the first threshold current $7.2 \mathrm{~mA}$, where PWD is the dominant mechanism for lengthening the bunch without increasing energy spread. Region-II, above the first threshold, shows the 1/3-power law similar to bunch lengthening. RegionIII, the regime above the second threshold, has also been observed at SPEAR [3], but is not well understood.

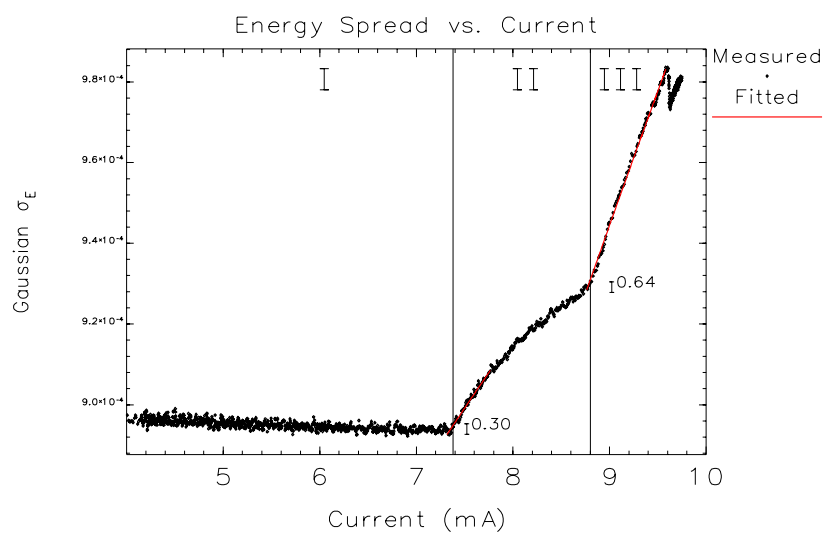

Figure 4: Bunch energy spread vs. current.

\subsection{Measurement without Dispersion Bump}

After our initial measurement, several girders were intentionally moved to reduce the background signal from the x-ray beam position monitor (BPM) [4]. In May 2000, when we simply re-installed the previously used setting of the dispersion-bump lattice, we could not store enough single-bunch current for the measurement. Instead of fully recovering the dispersion-bump lattice, we used the $\mathrm{S} 35: \mathrm{BM}$ source in the nominal lattice, where dispersion is reasonably large $(8 \mathrm{~cm})$. With the enhanced resolution of the x-ray pinhole camera (down to $22 \mu \mathrm{m}$ ), we measured bunch length and energy spread again as a function of current. Note that the camera resolution is much less than the beam size because of the energy spread. The results were essentially the same as those shown in Figs. 3 and 4 
except that the threshold was lowered slightly (1 mA). This decrease may be attributed to additional small gap ID chambers being installed in the ring, as well as the effect of the lattice change.

In addition to the static measurements, we also investigated the dynamic evidence of instability via the beam signal. We used the sum signal from the strip-line connected to a 26-GHz Hewlett Packard (HP) Vector Spectrum Analyzer (VSA). By setting the center frequency at $7.2 \mathrm{GHz}$, we measured the beam spectrum; the results are shown in Fig. 5.
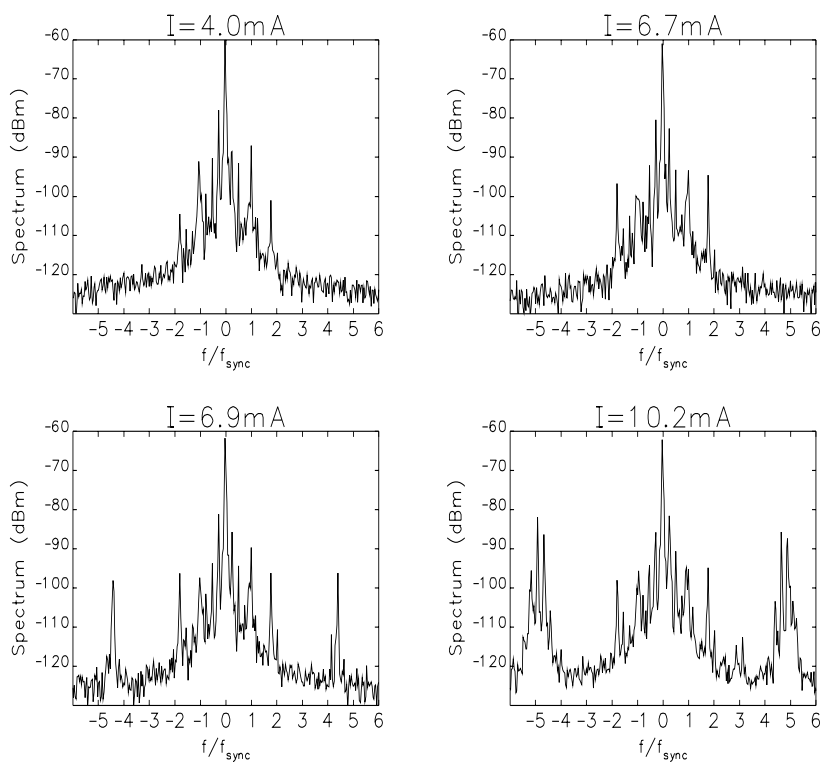

Figure 5: Beam spectra showing synchrotron sidebands for different current levels: 4.0, 6.7, 6.9, and 10.2 mA.

At low currents, we observed a coherent signal at $f_{s}$ $(m=1)$ and $2 f_{s}(m=2)$ where the measured synchrotron frequency is $\mathrm{f}_{\mathrm{s}}=1.412 \mathrm{kHz}$. The signal from dipole motion at $f_{s}$ usually does not change its frequency and strength as the current varies. In the APS storage ring, the typical amplitude of this motion is $0.5-1.0 \mathrm{ps}$. Up to $6.7 \mathrm{~mA}$, the spectra look the same, however, a strong coherent signal near $4 \mathrm{f}_{\mathrm{s}}(\mathrm{m}=4)$ appeared when the current increased to 6.9 $\mathrm{mA}$; here we also observed the energy spread begin to increase. In this measurement, the rf voltage was 7.5 MV instead of the nominal 9.4 MV.

On the basis of the static and dynamic measurements, we conclude that the instability threshold behavior we observed by a sudden increase in energy spread is related to a strong coherent motion within the bunch. This motion intensifies as the current increases, as evidenced by the spectrum at $10.2 \mathrm{~mA}$. This increased motion in turn results in the further increase in the energy spread.

Our findings are similar to the microwave instability described by Sacherer [5]. According to his theory, the blowup of bunch area is caused by the coupling of two modes. For our case, it seems that the modes coupled are $m=4$ and $m=5$. These are very high-order modes not observed in the lepton machine to the authors' knowledge.

\section{IMPEDANCE ESTIMATES}

The bunch length and the centroid time shift were measured at different rf voltages by using a streak camera. Image processing software fitted each density profile to a gaussian and produced an rms value and a time shift. We fitted the data as a function of current to the Haissinski solutions for bunch density using the simple $\mathrm{L}+\mathrm{R}$ model of longitudinal impedance in the form $\mathrm{Z}(\omega)=\mathrm{R}+\mathrm{j} \omega \mathrm{L}$.

We expect the $\mathrm{L}$ and $\mathrm{R}$ model components to fit roughly independently to the bunch length and bunch time shift, respectively. The agreement of the fit at low bunch length is expected to be less accurate because the bunch samples more complex geometrical impedances of the ring. Fig. 6 shows the bunch length data and the corresponding fitted lines for the best overall model fit of $\mathrm{Z} /\left.\mathrm{n}\right|_{0}=0.5 \Omega$ and $\mathrm{R}=3 \mathrm{k} \Omega$.

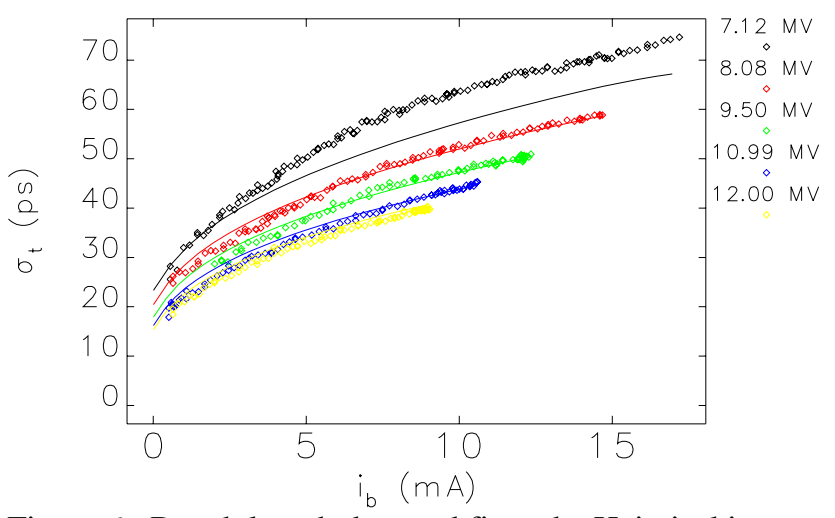

Figure 6: Bunch length data and fit to the Haissinski solution using $\mathrm{L}+\mathrm{R}$ impedance model.

\section{CONCLUSION}

We measured bunch length and energy spread as a function of currents in the APS storage ring. We observed that the threshold of longitudinal microwave instability occurred at 6-7 mA with rf voltage operating at 9.4 MV, above which energy spread increased and a coherent signal developed between $4 f_{s}$ and $5 f_{s}$. The fitting of bunch length data to the simple $\mathrm{L}+\mathrm{R}$ model estimated the impedance of the ring to be $0.5 \Omega$.

\section{REFERENCES}

[1] A.H. Lumpkin, et al., Nucl. Instrum. Methods A 393, 50 (1997).

[2] L. Emery, Proc. 1999 Part. Accel. Conf, 401 (2000).

[3] P.B. Wilson, et al., IEEE Trans. Nucl. Sci. 24, No. 3, 1211 (1977).

[4] G. Decker and O. Singh, Phy. Rev. ST-AB, Vol. 2, 112801 (1999).

[5] F.J. Sacherer, IEEE Trans. Nucl. Sci. 24, No. 3, 1393 (1977). 